\title{
CONHECIMENTO ATUAL SOBRE O COMPORTAMENTO ALIMENTAR DE COPÉPODOS CALANOIDA EM AMBIENTES MARINHOS
}

\author{
E. M. ESKINAZI-SANT'ANNA \\ ICB - Laboratório de Zooplâncton - UFMG \\ T. K. S. BJORNBERG \\ Centro de Biologia Marinha-USP
}

\begin{abstract}
RESUMO
Informações obtidas sobre estudos que abordam o comportamento alimentar de copépodos Calanoida foram reunidas, para traçar um panorama do conhecimento atual sobre o assunto. As informações atuais sugerem um padrão muito mais diversificado no comportamento alimentar dos copépodos do que o descrito nos primeiros estudos, incluindo a adoção de estratégias de alimentação e comportamento cognitivo na captura do alimento. Os estudos realizados sobre os hábitos alimentares de copépodos em águas costeiras brasileiras corroboraram estas idéias, mostrando que além de diatomáceas, detritos e protozooplâncton podem constituir importantes fontes adicionais de alimento, e que processos seletivos ocorrem na captura de alguns itens alimentares.

Palavras Chave: copépodos marinhos, alimento, comportamento alimentar
\end{abstract}

\begin{abstract}
A summary of information concerning copepod feeding behaviour was assembled, to provide a panorama about the current knowledge on this issue. Actually, information indicates that copepod feeding behaviour is much more diverse than described in earliers studies, including feeding strategies and cognitive behaviour during the food capture. Studies on the feeding behaviour of copepods from brazilian coastal waters support these findings, since it showed that besides diatoms, detritus and protozooplankton can also represent an important food resource, and that selective feeding occurs during the capture of some food items.

Key words: copepod, food, feeding behaviour
\end{abstract}

\section{INTRODUÇÃO}

A descrição das relações alimentares e da transferência de matéria e energia nos ecossistemas possibilita um maior conhecimento acerca dos complexos processos trofodinâmicos, auxiliando na elaboração de modelos funcionais mais realistas, que facilitem o manejo e gerenciamento dos sistemas naturais (WINEMILLER \& POLIS, 1996). Vários estudos têm sido realizados, visando conhecer com maior profundidade o comportamento alimentar de copépodos marinhos, especialmente em condições naturais (KlepPel et al., 1996; SAUTOUR \& CASTEL, 1999). No Brasil, existem poucos estudos enfocando o assunto, evidenciando a lacuna existente nesta área de pesquisa. Desta forma, algumas informações sobre o tema foram reunidas, com o objetivo de fornecer uma visão geral do conhecimento atual sobre o comportamento alimentar de copépodos, incluindo os resultados obtidos em estudos com copépodos de águas costeiras do Brasil. 


\section{METODOLOGIA}

As informações aqui reunidas foram obtidas na literatura disponível, não tendo sido estabelecida uma ordem cronológica para a citação das referências, uma vez que o principal objetivo desta revisão foi relatar o panorama atual de conhecimento sobre o comportamento alimentar de copépodos. Desta forma, foram descritas as primeiras informações sobre o assunto, as descobertas efetuadas e as transformações conceituais originadas a partir dos estudos sobre as relações tróficas de copépodos planctônicos.

\section{COMPORTAMENTO ALIMENTAR DE COPÉPODOS CALANOIDA} MARINHOS

Muitos estudos têm sido realizados com o objetivo de descrever a dieta dos copépodos e seu significado em termos de produção secundária (MARSHALL \& ORR, 1965; KLEPPEl et al., 1996, entre outros). O termo dieta, segundo KLEPPEL (1993), engloba tanto os aspectos quantitativos da alimentação ( $p$ e. taxa de ingestão), quanto os aspectos qualitativos (se autotrófica ou heterotrófica, tamanho, aspectos nutricionais, etc.). Os resultados dessas investigações têm mostrado que o tipo e a quantidade do alimento afetam diretamente a produção secundária de copépodos e consequentemente a produção em níveis tróficos mais elevados (Frost, 1972; SMITH \& EPPLEY, 1982; DURBIN et al., 1983; ROMAN, 1984). O tipo de alimento disponível pode ainda influenciar consideravelmente o deslocamento espacial, composição química, taxas de filtração e as estruturas filtradoras de populações de copépodos (ANRAKU \& OMORI, 1963; OMORI, 1973; PAFFENHÖFER, 1988; 1994; BAMSTEDT et al., 1999).

Vários experimentos de "grazing" in situ foram realizados, com o intuito de validar os resultados descritos em laboratório e também obter respostas sobre processos ainda não conhecidos completamente (HARDING, 1974; BAUTISTA \& HARRIS, 1992; Moloney \& GibBons, 1996; SAUtour \& CASTEL, 1999). Turner $(1985,1986)$, analisando o conteúdo alimentar de pelotas fecais de copépodos, encontrou que algumas espécies reconhecidamente herbívoras ou mesmo carnívoras podem na verdade, ser omnívoras, dependendo do tipo de alimento disponível, condição fisiológica do espécime e mesmo variabilidade física do ambiente. Os resultados obtidos em condições naturais têm servido de base para uma melhor avaliação das complexas condições alimentares aos quais os copépodos estão expostos, verificando-se que esta complexidade está refletida em suas dietas, e assim, em suas estratégias de alimentação (PRICE, 1988).

Segundo AlCARAZ et al. (1980) e STRICKLER (1982), além do simples fato de encontrar partículas alimentares, existe ainda uma componente cognitiva à alimentação, ou seja, os copépodos podem procurar ativamente por uma partícula, capturá-la e escolher entre ingerir ou rejeitá-la como uma fonte potencial de alimento. Se a alimentação pode ser seletiva, então o papel que os copépodos desempenham no ecossistema, é em parte, função das estratégias que eles utilizam para obter seu alimento (KLEPPEL, 1993). As estratégias de alimentação representam uma resposta dos copépodos ao ambiente, no qual o alimento encontra-se constituído por vários tipos de partículas, que refletem os processos dinâmicos característicos de cada sistema (LEGENDRE \& DEMERS, 1984; LEGENDRE \& RASSOUlZADEGAN, 1995). 
As estratégias de alimentação proporcionam a aquisição dos elementos nutricionais essenciais que podem estar escassos no ambiente, satisfazendo os requerimentos fisiológicos imprescindíveis ao desenvolvimento do organismo. Os requerimentos nutricionais de copépodos também têm sido amplamente discutidos, especialmente com relação à qualidade das partículas ingeridas, considerando a presença dos ácidos graxos poli-insaturados (PUFA). A variabilidade destes compostos nas partículas alimentares, considerados vitais para a produção de ovos e eclosão de náuplios, ainda precisa ser melhor estudada (CLAUSTRE et al., 1988; JÓNASDÓTTIR, 1994; KLEPPEL et al., 1998).

A questão da seletividade alimentar em copépodos vem sendo debatida há muito tempo (KLEPPEL, 1993). Até meados dos anos 70, acreditava-se que o processo de não-seletividade era dominante nos ambientes aquáticos. A filtração era considerada como o principal mecanismo para captura de alimento, embora processos raptoriais começassem a ser descritos (RICHMAN \& ROGERS, 1969). COWLES (1979), propôs que estratégias seletivas e não seletivas de alimentação poderiam ocorrer, de acordo com a disponibilidade alimentar no ambiente. $\mathrm{O}$ autor, ao estudar a alimentação de copépodos em águas ao largo do Peru, verificou que os espécimes apresentavam alimentação seletiva quando o alimento era abundante, e não-seletiva quando o alimento estava escasso.

Além dos estudos sobre os mecanismos utilizados na captura de alimento, tem havido um crescente interesse em relação aos aspectos cognitivos da alimentação. Resultados experimentais comprovaram a existência de percepção sensorial em copépodos (POULET \& MARSOT, 1978, entre outros), sendo que essas informações foram corroboradas quando os quimioreceptores, responsáveis pela discriminação do alimento no ambiente, foram descritos por FRIEDMAN \& STRICKLER (1975) e FRIEDMAN (1980). LEGIER-VISSER et al. (1986) descreveram modelos de detecção de presas através de direcionamento químico e estímulos táteis, que conduziam à ingestão ou rejeição de algumas partículas planctônicas. O papel dos quimioreceptores na seleção de partículas algais potencialmente tóxicas como dinoflagelados parece ainda bastante complexo, uma vez que alguns copépodos como Calanus tortanus e C. finmarchicus parecem rejeitar algumas espécies de dinoflagelados, mas são capazes de ingerir outras (HuNTLEY et al., 1986)

Nos ecossistemas marinhos, o elo direto entre as diatomáceas e os copépodos tem sido a descrição clássica das cadeias alimentares pelágicas. Diversos autores, entretanto, questionam esse padrão, baseados em novas descobertas sobre o papel funcional de organismos até então pouco estudados, como protozoários e bactérias (StOecker \& Egloff, 1987; StOeCKer \& CAPuzzo, 1990; AzAM et al., 1983). Segundo KLEPPEL et al., (1991), em alguns sistemas, as diatomáceas podem não representar a principal fonte alimentar para copépodos, já tendo sido observado que itens heterotróficos como dinoflagelados e microzooplâncton podem constituir-se em partículas alimentares preferenciais. Experimentos realizados pelo autor mostraram que a produção de ovos de Acartia tonsa foi fortemente afetada pela variabilidade na biomassa de dinoflagelados e microzooplâncton, não tendo sido encontrada nenhuma variação na produção de ovos relacionada à flutuabilidade da biomassa fitoplanctônica. Em outras cinco espécies de copépodos estudadas (Temora longicornis, Calanus helgolandicus, Centropages hamatus, Acartia clausi e Centropages furcatus) em 
ecossistemas distintos (águas costeiras, oceânicas e estuarinas), os resultados obtidos na análise do conteúdo digestivo e pigmentos mostraram uma predação preferencial sobre dinoflagelados e microzoooplâncton. A razão para essa preferência alimentar poderia ser a maior concentração de lipídios e proteínas em dinoflagelados do que em diatomáceas (HiTCHCOK, 1982). Ciliados também parecem ser mais ricos em proteínas e lipídios, do que o fitoplâncton (VERITY \& LANGDON, 1984).

Embora possam constituir o principal alimento na dieta de copépodos, alguns efeitos negativos de diatomáceas sobre o crescimento, produção e eclosão de ovos têm sido descritos. Entretanto, os mecanismos envolvidos nestes efeitos ainda não foram devidamente identificados (defensivos químicos, ausência de certos compostos bioquímicos, substâncias inibidoras de "grazing", substâncias tóxicas ou que afetem diretamente a fertilidade, desenvolvimento embrionário, viabilidade dos náuplios, etc) e nem se sabe ainda se estes mecanismos de defesa restringem-se a algumas espécies ( $p$. e. Phaeodactylum tricornutum, Thalassiosira rotula) ou são comuns ao grupo (MirAlto et al., 1995; IANORA et al., 1999).

Um novo desafio nos estudos sobre comportamento alimentar de copépodos tem sido ainda tentar explicar o aparente paradoxo de algumas espécies de diatomáceas apresentarem uma composição nutricional pobre, mas serem ingeridas em grande quantidade (BAN et al., 1997; JÓNASDÓTTIR et al., 1998).

Um outro fator extremamente importante ao se considerar a variação na dieta de copépodos refere-se à flexibilidade dessa dieta durante as diversas fases de desenvolvimento desses microcrustáceos. RofF et al., (1995), mostraram que náuplios e copepoditos podem desempenhar um papel fundamental como elo intermediário entre a teia alimentar microbiana e a clássica rede alimentar de pastagem, observando que náuplios de diversas espécies são capazes de bacterivoria. Essa constatação é especialmente importante em águas oceânicas, onde partículas bacterianas podem constituir a principal fonte alimentar. Nesses ambientes, a teia microbiana (formada pelo picoplâncton e bactérias heterotróficas) torna-se muito importante, já que pode ser capaz de sustentar as fases juvenis da teia alimentar clássica.

No Brasil, estudos abordando o comportamento alimentar in situ de copépodos são ainda bastante escassos. Alguns trabalhos considerando a alimentação de zooplâncton baseados na análise de isótopos estáveis foram desenvolvidos por MATSUURA \& WADA (1994) e SCHWAMBORN et al., (1999a). SCHWAMBORN (1997) e SCHWAMBORN et al., (1999b), estudaram a relação trófica entre zooplâncton e manguezais, relatando a assimilação de cerca de 13 a $40 \%$ do carbono oriundo de manguezal por Acartia sp, sob a forma de detritos. Um modelo quantitativo de simulação dos processos tróficos no fito e zooplâncton da área de ressurgência de Cabo Frio (RJ), foi realizado por CARBONEL \& VALENTIN, (1999).

Especificamente com relação a copépodos, LOPES (1997) obteve as primeiras informações sobre a atividade alimentar de Acartia lilljeborgi, Paracalanus quasimodo, Pseudodiaptomus acutus, Temora stylifera e T. turbinata na Baía de Paranaguá, através da quantificação de pigmentos do trato digestivo. Segundo o autor, não foi encontrada uma correlação entre a concentração ambiental de clorofila e a concentração no trato intestinal dos copépodos, sugerindo que processos hidrodinâmicos possam influenciar decisivamente no deslocamento espacial e nas estratégias de alimentação dos copépodos estudados. 
Estudos realizados por ESKINAZI-SANT'ANNA (2000), no Canal de São Sebastião (SP), mostraram que copépodos podem incluir uma variedade de itens alimentares em sua dieta básica. Análises do conteúdo alimentar das pelotas fecais de Parvocalanus crassirostris, Paracalanus quasimodo, Temora stylifera e Temora turbinata evidenciaram a ingestão de diatomáceas, dinoflagelados, detritos e do protozoário Sticholonche zanclea (Figura 1). A presença de S. zanclea nas pelotas foi registrada mesmo quando este protozoário apresentou densidades relativas bastante reduzidas no ambiente $(<10 \%)$, evidenciando a ocorrência de processos seletivos na captura deste item alimentar. Os fragmentos de diatomáceas identificados nas pelotas fecais mostraram ainda que também ocorreu uma ingestão seletiva do alimento algal, pois as espécies fitoplanctônicas identificadas nas pelotas não corresponderam às mais abundantes no ambiente.

Para a espécie Acartia lilljeborgi, (ESKINAZI-SANT'ANNA, em preparação), as análises das pelotas fecais mostraram que material amorfo, provavelmente de origem detrital, constituiu um item alimentar muito frequente, tendo sido observados em mais de $80 \%$ das pelotas fecais analisadas, enquanto que fragmentos de diatomáceas foram encontrados em poucas pelotas $(<20 \%$ do total analisado), representados basicamente por espinhos de Chaetoceros sp (Figura 2 ). A análise de pelotas fecais em microscopia eletrônica pode subestimar a presença de itens alimentares sem estruturas rígidas, mas constitui uma alternativa eficaz para estudos de relações tróficas. Uma abordagem complementar seria associar a análise de pelotas fecais com registros da alimentação in situ, através do estudo do conteúdo digestivo.

A obtenção de informações sobre o comportamento alimentar de copépodos em águas tropicais é extremamente importante, pois pode contribuir para a elaboração de modelos energéticos mais adequados a essa regiões. Considerando-se a variedade de ecossistemas marinhos no Brasil e a diversidade de espécies de copépodos, pode-se visualizar o vasto campo de investigação a ser abordado, considerado por BRANDINI et al., (1997), como um enfoque prioritário a ser explorado em estudos futuros, representando assim uma nova perspectiva nos estudos ecológicos das comunidades planctônicas no Brasil.

\section{AGRADECIMENTOS}

À Dra. Arnola Cecília Rietzler pelas sugestões ao manuscrito e à FAPESP, pelo apoio financeiro (Proc. 96/9774-4). Ao revisor, pela leitura cuidadosa e sugestões.

\section{REFERÊNCIAS}

AlCARAZ, M. PAFFenhöFer, G.-A.; StRICKLER, J.R. (1980). Catching the algae: a first account of visual observations on filter-feedings calanoids. In: KERFOOT, W.C. (ed). Evolution and Ecology of Zooplankton Communities. University Press of New England, Hanover, 560p.

ANRAKU, M.; OMORI, M. (1963). Preliminary survey of the relationship between the feeding habitat and the structure of the mouth-parts of marine copepods. Limnol. Oceanog., 8: 116-126.

AzAm, F.T.; Fenchel, T.; Field, J.G.; Meyer-Reil, L.A.; Thingstad, F. (1983). The ecological role of water column microbes in the sea. Mar. Ecol. Prog. Ser., 10: 257-263. 
Bamstedt, U.; NejStgaArd, J.C.; SolberG, P.T. (1999). Utilization of small-sized food algae by Calanus finmarchicus (Coepoda, Calanoida) and the significance of feeding history. SARSIA, 84: 19-38.

Ban, S.; Burns, C.; Castel, J. Christou, E. Escribano, R. Fonda-Umani, S.; Gasparini, S.; Guerrero Ruiz, F. Hoffmeyer, M.; IANORA, A.; Kang H.; Laabir, M. Lacoste, A. Miralto, A. Poulet, S.; Ning, X.; Rodriguez, V.; Runge, J. Shi, J. Starr, M. Uye, S., WANG, Y. (1997). The paradox of diatomcopepod interactions. Mar. Ecol. Prog. Ser., 157: 287-293.

BAUTISTA, B. HARRIS, R.P. (1992). Copepod gut contents, ingestion rates and grazing impact on phytoplankton during a spring bloom. Mar. Ecol. Prog. Ser., 82: 41-50.

BRANDINI, F.P.; LOPeS, R.M.; GUTSEIT, K.S.; SPACH，H.L.; SASSI, R. (1997). Planctonologia na plataforma continental do Brasil. Diagnose e revisão bibliográfica. MMA, CIRM, FEMAR 196p.

Claustre, H. Marty, J.C.; Cassiani, L. Dagaut, J. (1988). Fatty acid dynamics in phytoplankton and microzooplankton communities during a spring bloom in the coastal Ligurian Sea. Mar. Microd. Food Webs, 3: 51-66.

COWLES, T.J. (1979). The feeding response of copepods from the Peru upwelling system: food size selection. J. Mar. Res., 13: 601-622.

DurBIN, E.G.; DuRBIN, A.G.; SMAYDA, T.J.; VERITY, P.G. (1983). Food limitation of production by adult Acartia tonsa in Narragansett Bay, Rhode Island. Limnol. Oceanogr., 28: 1199-1213.

EskinaZi-Sant'Anna, E.M. (2000). Estudo da dieta natural de Parvocalanus crassirostris, Paracalanus quasimodo, Temora stylifera e Temora turbinata (Copepoda, Calanoida), no Canal de São Sebastião (SP, Brasil). São Paulo, 317p. Tese de Doutorado. Universidade de São Paulo.

FRIEDMAN, M.M.; STRICKLER, J.R. (1975). Chemoreceptores and feeding in calanoid copepods (Arthropoda: Crustacea). Proc. Natl. Acad. Sci., 72: 4185-4188.

FRIEDMAN, M.M. (1980). Comparative morphology anf functional significance of copepods receptores and oral structures. In: KERFOOT, W.C. (ed). Evolution and ecology of zooplankton communities. University of Hampshire, Hanover, p. 185197.

Frost, B.W. (1972). Effects of food particles on the feeding behavior of the marine planktonic copepod Calanus pacificus. Limnol. Oceanogr., 17: 805-815.

HARDING, G.C.H. (1974). The food of deep-sea copepods. J. mar. biol. Ass. U.K., 54: 141-155.

HiтсHCOCK, G.L. (1982). A comparative study of the size-dependent organic composition of marine diatoms and dinoflagellates. J. Plankton Res., 4: 363-377.

Huntley, M.E.; Sykes, P.; Rohan, S.; MARIN, V. (1986). Chemically mediated rejection of dinoflagellate prey by the copepods Calanus pacificus and Paracalanus parvus: mechanisms, occurrence, significance. Mar. Ecol. Prog. Ser., 28: 105-120.

Ianora, A.; Miralto, A.; Poulet, S.A. (1999). Are diatoms good or toxic for copeods? reply to comment by Jónasdóttir et al. Mar. Ecol. Prog. Ser.,177: 305308 .

JÓNASDÓTTIR, S.H. (1994). Effects of food quality on the reproductive sucess of Acartia tonsa and Acartia hudsonica: laboratory observations. Mar. Biol., 121: 67-81. 
JÓNASDÓTTIR, S.H.; KIORBOE, T.; TANG, K.W.; JOHN, M.S.; VISSERR, A.W.; SAIZ, E.; DAM, H.G. (1998). Role of diatoms in copepod production: good, harmless or toxic? Mar. Ecol. Prog. Ser.,172: 305-308.

KLEPPEl, G.S.; HollidAY, D.V.; PIEPER, R.E. (1991). Trophic interactions between copepods and microplankton: a question about the role of diatoms. Limnol. Oceanogr., 36: 172-178.

KLePpel, G.S. (1993). On the diets of calanoid copepods. Mar. Ecol. Prog. Ser., 99: 183-195.

Kleppel, G.S.; Burkart, C.A.; CARTer, C.; Tomas, C. (1996). Diets of calanoid copepods on the West Florida continental shelf: relationships between food concentration, food composition and feeding activity. Mar. Biol., 127: 209-217.

KLEPPEL, G.S.; BURKART, C.A.; HouChIN, L. (1998). Nutrition and regulation of egg production in the calanoid copepod Acartia tonsa. Limnol. Oceanogr.,43: 10001007.

LEGENDRE, L.; DEMERS, S. (1984). Towards dynamic biological oceanography and limnology. Can. J. Fis. Aquat. Sci., 41: 2-19.

LEGENDRE, L.; RASSOUlZADEGAN, F. (1995). Plankton and nutrient dynamics in marine waters. OPHELIA, 41: 153-172.

LeGier-Visser, M.F.; Mitchell, J.G.; OKubO, J.G.; Furhman, J.A. (1986). Mechanoreception in calanoid copepods. A mechanism for prey detection. Mar. Biol., 90: 529-535.

LOPES, R.M. (1997). Distribuição espacial, variação temporal e atividade alimentar do zooplâncton no complexo estuarino de Paranaguá. Curitiba, 140p. Tese de Doutorado. Universidade Federal do Paraná.

MARShall, S.M.; ORR, A.P. (1965). The biology of a marine copepod, Calanus pacificus (Gunnerus). Oliver and Boyd, London, 345p.

MATSUURA, Y.; WADA, E. (1994). Carbon and nitrogen stable isotope ratios in marine organic matters of the coastal ecosystem in Ubatuba, southern Brazil. Ciência e Cultura, 46: 141-146.

Mauchline, J. (1998). The Biology of Calanoid Copepods. Advances in Marine Biology, Academic Press, London, 710p.

Miralto, A.; IAnora, A. Poulet, S. A. (1995). Food type induces different reproductive responses in the copepod Centropages typicus. J. Plankton Res., 17: 1521-1534.

Moloney, C.L.; GibBons, M.J. (1996). Sampling and analysis of gut contents in relation to environmental variability and diel vertical migration by herbivorous zooplankton. J. Plankton Res., 18: 1535-1556.

OMORI, M. (1973). Variations of lenght, weight, respiration rate, and chemical composition of Calanus cristatus in relation to its food and feeding. In: STEELE, J.H. (Ed). Marine Food Chains. Olivier \& Body, London, 551p.

PAFFENHÖFER, G.-A. (1988). Feeding rates and behaviour of zooplankton. Bull. Mar. Sci., 43 (3): 430-455.

PetiPA, T.S.; Pavlova, E.V.; Mironov, G.N. (1973). The food web structure, utilization and transport of energy by trophic levels in the planktonic communities. Em: Steele, J.H. (Ed). Marine Food Chains. Olivier \& Body, London, 551p. 
Poulet, S.A.; MARSOT, P. (1978). Chemosensory grazing by marine calanoid copepods (Arthropoda: Crustacea). Science, 200:1403-1405.

PRICE, H.J. (1988). Feeding mechanisms in marine and freshwater zooplankton. Bull. Mar. Sci., 43 (3): 327-343.

Richman, S.; Rogers, J.N. (1969). The feeding of Calanus helgolandicus on synchronously growing populations of the marine diatom Ditylum brightwelli. Limnol. Oceanogr., 14: 701-709.

RofF, J.C.; TURner, J.T.; WebBer, M.K.; HopCroff, R.R. (1995). Bacterivory by tropical copepod nauplii: extent and possible significance. Aquat. Microbiol. Ecol., 9:165-175.

Roman, M.R. (1984). Utilization of detritus by the copepod Acartia tonsa. Limnol. Oceanogr., 29: 949-959.

SAUTOUR, B.; CASTEL, J. (1999). Grazing activity of mesoplanktonic copepods in a shallow bay during an algal spring bloom (Marennes-Oléron Bay, France). J. Mar. Biol. Ass. U.K., 79: 73-84.

SCHWAMBORN, R. (1997). Influence of mangroves on community structure and nutrition of macrozooplankton in Northeast Brazil. ZMT Contribution. (Bremen). 4: 1-77.

Schwamborn, R.; Voss, M.; Ekau, W.; SAInT-PAul, U. (1999a). Stable isotope compostion of particulate organic matter and zooplankton in North-East Brazilian shelf waters. Arch. Fish. Mar. Res., 47: 201-210.

Schwamborn, R.; EKau, W.; Silva, A.P.; Silva, T.A.; SainT-Paul, U. (1999b). The contribution of estuarine decapod larvae to marine zooplankton communities in North-East Brazil. Arch. Fish. Mar. Res., 47: 167-182.

SMITH, P.E.; EPPlEY, R.W. (1982). Primary production and the anchovy population in the Southern California Bight: comparison of time series. Limnol. Oceanogr., 27:1-17.

Stoecker, D.K.; Egloff, D.A. (1987). Predation by Acartia tonsa Dana on planktonic ciliates and rotifers. J. Exp. Mar. Biol. Ecol., 110, 53-68.

Stoecker, D.K.; CAPuzzo, J.M. (1990). Predation on protozoa: its importance to zooplankton. J. Plankton Res., 12: 891-908.

STRICKLER, J.R. (1982). Calanoid copepods, feeding currents and the role of gravity. Science, 218: 158-160.

TURNER, J.T. (1985). Zooplankton Feeding Ecology: Contents of Fecal Pellets of the Copepod Anomalocera ornata from Continental Shelf and Slope Waters of the Gulf of Mexico. Mar. Ecol., 6 (4): 285-298.

TURNER, J.T. (1986). Zooplankton Feeding Ecology: Contents of Fecal Pellets of the Copepod Undinula vulgaris from Continental Shelf Waters of the Gulf of Mexico. Mar. Ecol., 7 (1): 1-14.

TURnER, J.T.; RofF, J.C. (1993). Trophic Leves and Trophospecies in Marine Plankton: Lessons from the Microbial Food Web. Mar. Microb. Food Webs, 7 (2): 225-248.

VALENTIN, J.L.; CARBONEL, M. (1999). Numerical modelling of phytoplankton bloom in the uupwelling ecosystemof Cabo Frio (Brazil). Ecol. Model., 116: 135-148.

VERITY, P.G.; LANGDON, C. (1984). Relationships between lorica volume, carbon, nitrogen and ATP content of tintinnids in Narragansett Bay. J. Plankton Res., 6: 859-868.

Winemiller, K.O.; Polis, G.A. (1996). Food Webs: What Can They Tell Us About the World? In: Polis, G.A.; Winwmiller, K.O. (eds). Food webs. Integration of Patterns and Dynamics. Chapman \& Hall, New York, 472p. 


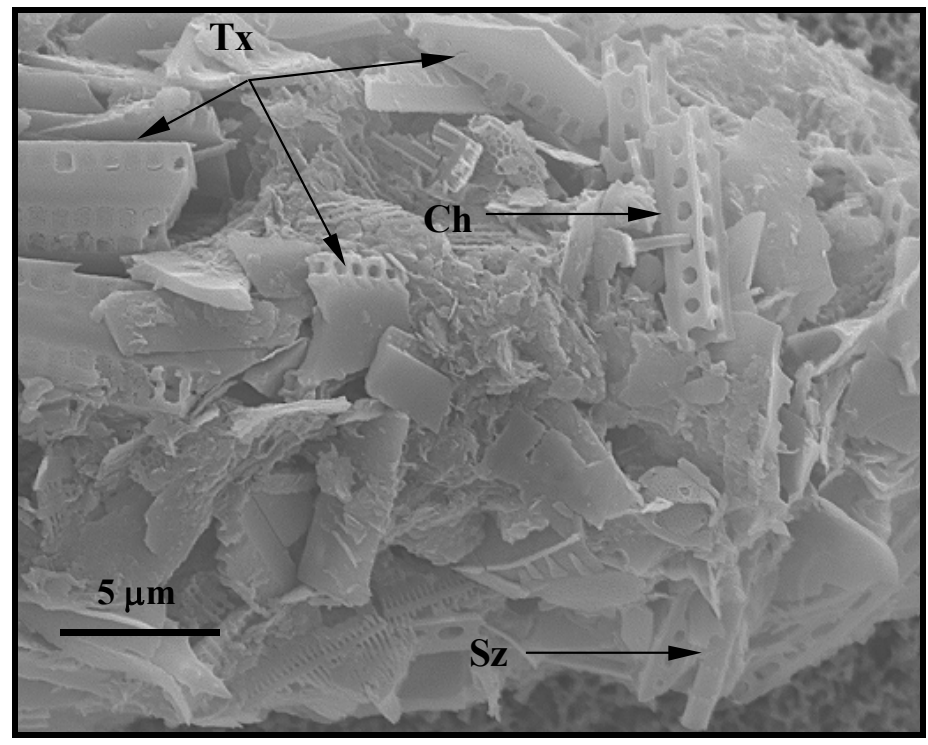

(A)

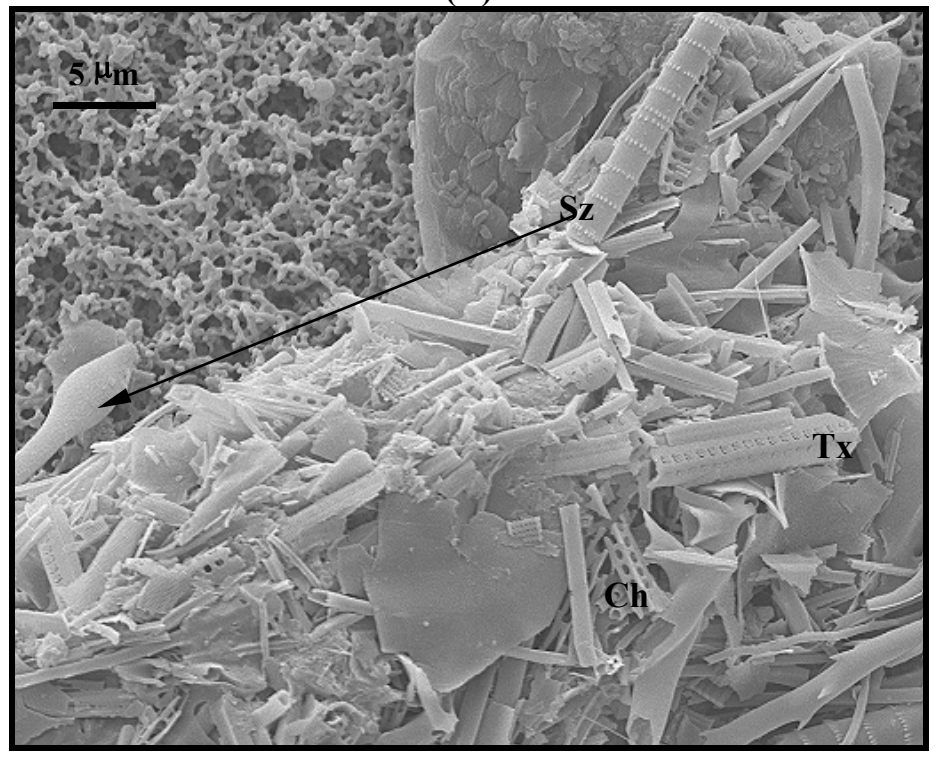

(B)

Figura 1. (A). Conteúdo alimentar das pelotas fecais de Parvocalanus crassirostris. Fragmentos de Thalassiotrix frauenfeldii (Tx), Chaetoceros sp (Ch) e Sticholonche zanclea (Sz). 3960x. (B). Conteúdo alimentar das pelotas fecais de Paracalanus quasimodo. Fragmentos de Thalassiotrix frauenfeldii (Tx), Chaetoceros sp (Ch) e Sticholonche zanclea (Sz). 2520x. Segundo ESKINAZI-SANT'ANNA (2000).

Trab. Oceanog. Univ. Fed. PE, Recife, 28(1):11 - 20, 2000. 


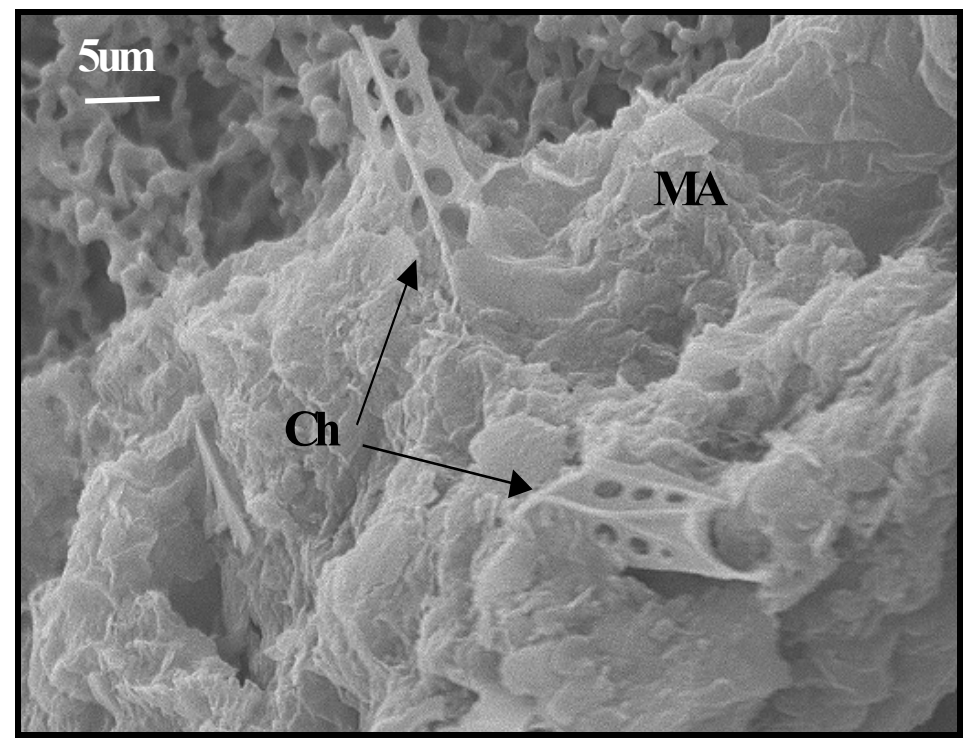

(A)

Figura 2. (A). Conteúdo alimentar das pelotas fecais de Acartia lilljeborgi. Fragmentos Chaetoceros sp (Ch) e Material Amorfo (MA) 3600x. 15kV. Segundo ESKINAZI-SANT’ANNA (em preparação). 